\title{
Applying a methodology for the design, delivery and evaluation of learning resources for remote experimentation
}

\author{
Alexander Mikroyannidis*, John Domingue*, Daan Pareit ${ }^{\dagger}$, Jono Vanhie-Van Gerwen ${ }^{\dagger}$, Christos Tranoris ${ }^{\S}$, Guillaume Jourjon ${ }^{\Uparrow}$, \\ and Johann M. Marquez-Barjall \\ ${ }^{*}$ The Open University, United Kingdom; ${ }^{\dagger}$ iMinds, Belgium; ${ }^{\S}$ University of Patras, Greece; ${ }^{\natural}$ Data61-CSIRO, Australia; $\|$ Trinity \\ College Dublin (TCD), Ireland.
}

\begin{abstract}
Remote labs and online experimentation offer a rich opportunity to learners by allowing them to control real equipment at distance in order to conduct scientific investigations. Remote labs and online experimentation build on top of numerous emerging technologies for supporting remote experiments and promoting the immersion of the learner in online environments recreating the real experience. This paper presents a methodology for the design, delivery and evaluation of learning resources for remote experimentation. This methodology has been developed in the context of the European project FORGE, which promotes online learning using Future Internet Research and Experimentation (FIRE) facilities. FORGE is a step towards turning FIRE into a pan-European educational platform for Future Internet. This will benefit learners and educators by giving them both access to world-class facilities in order to carry out experiments on e.g. new internet protocols. In turn, this supports constructivist and self-regulated learning approaches, through the use of interactive learning resources, such as eBooks.
\end{abstract}

Keywords-Remote experimentation; Interactive learning resources; Widgets; Open Educational Resources; Massive Open Online Courses, Future Internet

\section{INTRODUCTION}

Higher education is currently undergoing major changes, largely driven by the availability of high quality online materials, also known as Open Educational Resources (OERs). OERs can be described as "teaching, learning and research resources that reside in the public domain or have been released under an intellectual property license that permits their free use or repurposing by others depending on which Creative Commons license is used" [1]. The emergence of OERs has greatly facilitated online education (eLearning) through the use and sharing of open and reusable learning resources on the Web. Learners and educators can now access, download, remix, and republish a wide variety of quality learning materials available through open services provided in the cloud.

The OER initiative has recently culminated in MOOCs (Massive Open Online Courses) delivered via providers such as Udacity ${ }^{1}$, Coursera ${ }^{2}$ and $\mathrm{edX}^{3}$. MOOCs have very quickly

${ }^{1}$ http://www.udacity.com/

2 https://www.coursera.org/ attracted large numbers of learners; for example over 400,000 students have registered within four months in $\mathrm{edX}^{4}$. Also, in the four years since the Open University started making course materials freely available in Apple's iTunes U, nearly 60 million downloads have been recorded worldwide ${ }^{5}$. More recently, the Open University established FutureLearn ${ }^{6}$ as the UK response to the emergence of MOOCs, in collaboration with premier British institutions such as the British Council, the British Library and the British Museum.

These initiatives have led to widespread publicity and also strategic dialogue in the education sector. The consensus within education is that after the Internet-induced revolutions in communication, business, entertainment, media, amongst others, it is now the turn of universities. Exactly where this revolution will lead is not yet known but some radical predictions have been made including the end of the need for university campuses ${ }^{4}$, while milder future outlooks are discussing 'blended learning' (combination of traditional lectures with new digital interactive activities). The consensus is however that the way higher education students learn is about to change radically.

The Future Internet Research and Experimentation (FIRE) ${ }^{7}$ initiative has been established to ensure that the European Internet Industry evolves towards a Future Internet containing European technology, services and values. So far, it is primarily used by academic and industry professionals for research and development of new Internet technologies, rather than being used for educational purposes. Through FIRE, a variety of facilities have been developed, including cloud computing services, 4G/5G experimental networks, cognitive radio networks, Wi-Fi and sensor network testbeds, infrastructures for High Performance Computing, and more. However, the corresponding costs both for the establishment and operation of these facilities is not to be neglected. Therefore, optimal usage

\footnotetext{
${ }^{3}$ https://www.edx.org/

${ }^{4}$ http://www.guardian.co.uk/education/2012/nov/11/online-freelearning-end-of-university

${ }^{5}$ http://projects.kmi.open.ac.uk/itunesu/impact/

${ }^{6}$ http://www.futurelearn.com/

${ }^{7}$ http://www.ict-fire.eu
} 
of the FIRE facilities is desired by its owners, a goal which has not been yet achieved.

This paper presents a methodology for the development of open interactive courseware, aimed at fostering remote experimentation. The methodology has been developed in the context of the FORGE project ${ }^{8}$ [2], which is a European initiative for online learning and experimentation via interactive learning resources. FORGE provides learners and educators with access to world-class experimentation facilities and high quality learning materials via a rigorous production process. In FORGE, we focus on remote experimentation performed on top of FIRE facilities. FORGE is specifying development methodologies and best practices for offering open courseware and online experimentation facilities to learners, related primarily to communications and IT.

The remainder of this paper is organised as follows. Section II introduces the methodology of the FORGE project and discusses its main principles regarding the establishment of a technological and pedagogical framework for remote labs and online experimentation. Section III presents the overall architecture of the FORGE infrastructure for building remote labs and delivering them to students. The application of the FORGE methodology for the design, delivery and evaluation of a real-life postgraduate course is presented in Section IV. Finally, the paper is concluded and the next steps of this work are discussed in Section V.

\section{THE FORGE METHODOLOGY}

One of the main goals of FORGE is to enable educators and learners to access and actively use FIRE facilities in order to conduct scientific experiments. We thus follow a constructivist approach to education where learning takes place by students creating artefacts rather than assuming the passive role of a listener or reader. Our approach is based on a wide range of studies that have shown that with the right scaffolding competent learners benefit greatly from constructivist or learning-by-doing approaches [3-5]. The experiment-driven approach of FORGE contributes to fostering constructivist learning by turning learners into active scientific investigators, equipped with world-class experimentation facilities.

From a learning technology perspective, FORGE is building upon new trends in online education. More specifically, in online educational platforms such as iTunes U, as well as in MOOCs, we see the large-scale take-up and use of rich media content. These include video in a variety of formats including webcasts and podcasts and eBooks, which can contain multimedia and interactive segments. In particular, eBooks provide a new level of interactivity since specific learning text, images and video can be closely integrated to interactive exercises ${ }^{9}$. In the context of the European project EUCLID $^{10}$ (EdUcational Curriculum for the usage of LInked Data), we have been producing such interactive learning resources about Linked Data and delivering them in a variety

\footnotetext{
${ }^{8}$ http://ict-forge.eu

${ }^{9}$ http://www.youtube.com/watch?v=KXCHKYsilq8

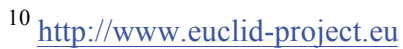

of formats, in order to be accessed from a variety of devices, both mobile (tablets and smartphones), as well as desktop computers. Building on this work, FORGE is producing interactive learning resources targeting a wide range of mediums and devices in order to maximise its impact on the eLearning community.

FORGE is enabling students to set-up and run FIRE experiments from within rich related learning content embedded as widgets inside interactive learning resources. Widgets are powerful software components that can be reused across different learning contexts and for different educational purposes. They offer a simple interface and can accomplish a simple task, such as displaying a news feed. They can also communicate with each other and exchange data, so that they can be used together to create mashups of widgets that complement each other. The portability of widgets as bespoke apps that can be embedded into a variety of online environments ensures that the FORGE learning solutions implemented as widgets have a high reusability factor across multiple learning domains and online learning technologies. Within FORGE, widgets enable educators and learners to access and actively use Future Internet facilities as remote labs in order to conduct scientific experiments. Learners and educators can setup and run Future Internet experiments from within rich related learning content embedded as widgets inside interactive eBooks and Learning Management Systems (LMSs) or Virtual Learning Environments (VLEs).

There are different available methodologies for deploying remote and/or virtual lab/courses in the literature, each of them targeting different facilities and tools. Bose [6] presents a methodology for creation of a virtual lab. This approach targets the Virtual labs project and guides the lab creator to deploy a lab within the scope of such project. In a similar basis, Frerich et al [7] present a lab lifecycle with the Excellent Teaching and Learning in Engineering Sciences (ELLI) project. Both mentioned projects, focus on virtual labs (software/simulationbased) rather than remote experimentation labs (experimentation-based) [8]. Additionally, the European project Go-Lab ${ }^{11}$ focuses on virtual labs in the context of inquiry-based learning. Compared to FIRE, the Go-Lab virtual labs are simpler as they are mainly targeted to Secondary Education. On the other hand, FORGE is primarily concerned with Higher Education, as it employs the more complex experimental setups offered by FIRE.

The FORGE methodology for the production of FIREenabled courseware consists of the following steps:

- $\quad$ Specifying course requirements. In this step, the educator specifies the overall course requirements, including the learning objectives of the course, the required skills, the skills that will be acquired by learners after completing this course, the course timeframe, the number of learners and the method of delivery (online, face-to-face, or blended).

- Identifying FIRE facilities. In this step, the educator identifies the FIRE facilities that will suit the course requirements. These FIRE facilities will be selected based

${ }^{11}$ http://www.go-lab-project.eu 
on their suitability for the learning objectives of the course and its associated skills. The number of learners and timeframe will also play a role in selecting a FIRE facility based on its availability. The first and most important task is to identify the facility features, which match the intended course content. When someone, for example, wants to include experimental exercises using specially developed wireless transmission protocols, a facility should be chosen where one has permission to adapt the radio drivers or where one can use cognitive radio devices, etc. A basic overview of the most prominent facility features covered in Fed4FIRE ${ }^{12}$ portal.

- Authoring educational content. The educational content that will form the learning pathway of the course is authored in this step. Finding open educational resources that are suitable for the course is quite important, as these can be reused, adapted and repurposed to fit the course learning objectives and other requirements. These resources can have the form of text that describes the theory behind a specific exercise, questionnaires with multiple-choice options, videos with lectures, videos with instructions on how to conduct the exercise, images and diagrams about the architecture and topology of the required components, graphical representations of the desired results etc.

- Integration of FIRE facilities and content. In this step, the selected FIRE facilities and the educational content of the course are integrated in order to form the complete learning pathway. FIRE facilities are commonly integrated as widgets, which can be reused across different learning activities for different learning purposes.

- Deployment. The deployment of the course for delivery to learners is performed in this step. Depending on the course requirement for delivery (online, face-to-face, or blended), the educator can deploy the course within a Learning Management System (LMS), a Virtual Learning Environment (VLE), or as an interactive eBook.

- Evaluation. In this step, the educator evaluates the success of the course, based on qualitative feedback received from learners via surveys and questionnaires, or via quantitative data collected by Learning Analytics tools that track the interactions of learners with the course materials and with each other.

- Reflection and adaptation. By analysing the qualitative and quantitative data collected from the evaluation of the course, educators have the opportunity to reflect and draw some conclusions not only about potential adaptations and improvements to the course, but also, and most important, on the impact of the course on the students and their skills and knowledge acquired.

Figure 1 summarizes the FORGE methodology, showing the steps to be followed in order to deploy, create, use, and/or reuse a FORGE course. As depicted, two main phases should be considered: a) Course preparation, and b) Course

${ }^{12} \mathrm{http}: / / w w w . f e d 4$ fire.eu deployment. At each of those phases, different processes should come into play. This paper provides a description of such processes and aims to guide course developers and learners towards a successful course deployment and learning experience.

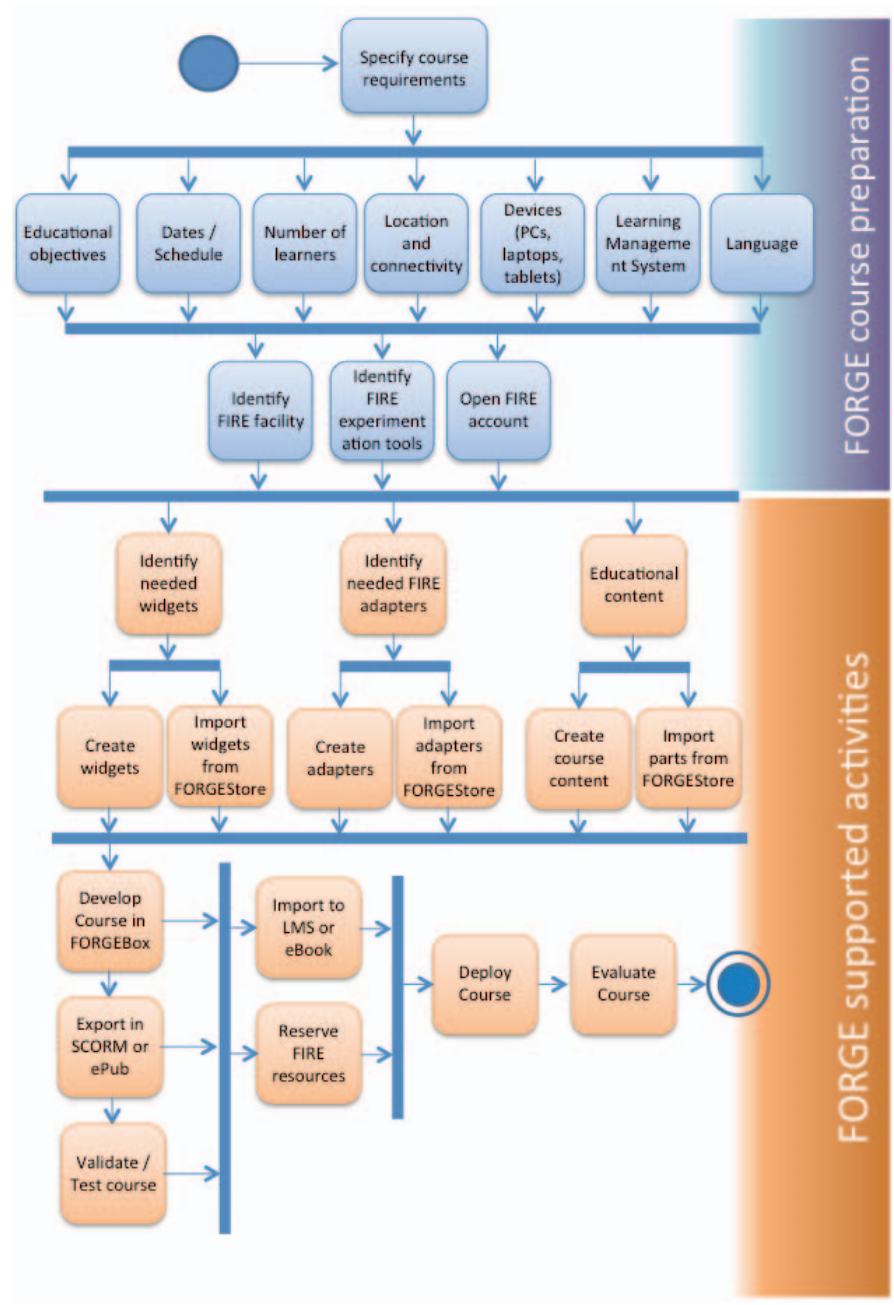

Figure 1: The FORGE methodology flowchart.

\section{THE FORGE REFERENCE ARCHITECTURE}

Key elements for supporting remote experimentation are the interactive elements that learners use while interacting with remote experimentation resources. We call these interactive elements widgets. These are consumable web applications that are hosted in a web server capable of supporting the interaction with remote resources. Widgets are also bound with services that implement, control and handle the communication with every remote experimentation resource. These backend services are also referred to as 'FIRE Resource Adapters'. Next, when we refer to widgets, we refer to this combination of web content and backend support for remote interactivity.

Figure 2 displays our proposed reference architecture for a widget, with architectural components that a developer would need to implement in order to achieve the best desirable result of bridging learning with remote resource interactivity. Since widgets are web services hosted somewhere on the web, ready 
to be consumed by other web content, the architecture defines both the widget UI as well as the backend domain logic and core architectural components.

Different functionality is defined in a way that a widget should respond differently depending on the user role: Learner, Teacher/Instructor, LMS/VLE administrator, widget service administrator. The generic architecture defines also the technologies that should be used to best perform the desired result, ease of use and effortless integration with existing Learning environments. Thus we propose the usage of LTI $2.0^{13}$ for best integration as well as Learning Analytics repositories for monitoring user behaviour while experiencing the lab course and interacting with the experimentation resources. The following paragraphs present these concepts in more detail.

\section{A. Supported roles by widgets and equivalent frontend interfaces}

The proposed architecture tries to support the following user roles:

- Widget Service administrator: Service Administrator is the user responsible for the whole widget web service. Service Administrator can login to the host machine and administer the service that provides the widget to consumers. Service Administrator can also manage for example users, registrations etc. The use cases are specific to the capabilities that the widget service will offer. E.g. the administrator of the ssh2web widget can allow specific domains that can use the service.

- LMS/VLE administrator: This user is the one responsible to integrate the widget to the target learning system LMS/VLE or even in an eBook. He needs to pay attention to the widget documentation, how it is delivered (i.e. as a URL), its API, its LTI compatibility, etc. For example, an administrator responsible for a Moodle installation could visit FORGEStore and read the documentation of the widget. Then he could register the widget into the Moodle environment by using the LTI registration URL of the widget service

- Teacher/Instructor: This user will define the behaviour and settings for a specific course. He can also use the interface to reserve resources or setup the testbed.

- Learner: This user will interact with the widget and the remote resource during the learning process.

The widgets UI layer defines components that will support the requirements of these roles. A widget UI is the main component that a user uses to interact with the widget. To behave correctly, the Widget service must know the context that it works under, in order to properly display the equivalent UI according to the user role. Thus, if possible, the widget should be aware of:

- The consumer service into which it is hosted and operating (i.e. is it an LMS/VLE, the VLE URL, an eBook, etc.);

\footnotetext{
${ }^{13}$ http://www.imsglobal.org/lti/
}

- The kind of consumer (i.e. its capabilities, browser, tablet etc.);

- The identity of the current user and his role

- The current course (content or page reference).

All this information can be passed either through a widget API (e.g. passing URL parameters, RESTful service, etc.) or via more education oriented ways like the LTI API. According to the user role there should be different UIs. It is not necessary for widgets to implement all these user interfaces. For example, the FORGE widgets of Teacher Companion Lab courses don't need to provide a Learner UI since they can only be used by teachers.

\section{B. Backend widget services}

The backend of a widget contains all the support services that implement all the widget's domain logic. The core services that are envisaged by FORGE widget reference architecture are presented in the next sections.

\section{1) Authentication Authorization Identity}

This service handles the users accessing the widget service, while affecting the widget behaviour according to user role (i.e. provide the equivalent user interface). What is interesting to provide is an LTI2.0 implementation. This will allow, as discussed before, the better integration of the widget with existing VLEs. Thus we recommend widget developers implementing a bridge service between the AAI widget service and an LTI 2.0 support library.

\section{2) User activity monitoring}

This service is responsible for monitoring user activity while interacting with a facility, especially if the widget needs to audit users for their behaviour (i.e. using Learning Analytics). What would be useful to implement is to provide to teachers the user behaviour while interacting with the facility. So it is highly recommended for widget developers to consider the integration of the Experience API (xAPI) and the ability to report user behaviour to an external Learning Record Store (LRS).

\section{3) FIRE Resource adapter}

FIRE adapters are tightly integrated with a widget, since the widget will provide the UI interacting with the FIRE facility. FIRE adapters will use provided APIs and tools from FIRE, especially those developed by the Fed4FIRE project which is providing harmonized APIs and tools across most FIRE facilities. The functionality of FIRE Adapters is described in more detail in the FORGE project deliverables ${ }^{14}$.

\section{ApPlying The FORGE Methodology And REFERENCE ARCHITECTURE}

The FORGE methodology and reference architecture has been successfully applied for a WLAN lab within a postgraduate course on wireless communication technologies. The developed lab session explains Wi-Fi throughout concepts

${ }^{14} \mathrm{http}$ ://ict-forge.eu/wp-content/uploads/2015/02/FORGE-2014-PD21-Final.pdf 
via a set of interactive exercises using learning resources for remote experimentation. The different steps of the FORGE methodology have been applied thoroughly, resulting in the creation of a lab where learning resources (including dedicated high-end hardware resources) are reserved and fully configured with only a few clicks on a web interface. By following all steps of the methodology, the lab includes various e-learning features (e.g. Learning Analytics) and can be integrated in any media (e.g. in an LMS/VLE, eBook, or a standalone web site). The creation and execution of this lab, using the aforementioned methodology and architecture is described in the next subsections.

\section{A. Applying the FORGE methodology}

The FORGE methodology for the production of this WLAN lab was applied using the steps of section II:

Specifying course requirements. The objective of the WLAN lab is for students to gain a deep understanding of the access mechanism in a Wi-Fi network after they have first acquired the theoretical knowledge via traditional lectures and exercise lessons. Learning outcomes of the lab with remote experimentation are the ability to explain the impact of different parameters (configuration mode, packet size, physical data rate, Quality of Service settings etc.) on the achievable wireless throughput in these networks. The WLAN lab is intended to be included in a master course on wireless technologies for students with background knowledge on basic networking protocols. The lab is in the first instance aimed at being taught within a course on wireless networking at a specific Belgian university for about 90 students each year. Nevertheless, the lab is intended to be reused in similar courses by other universities or institutions as well. The time for the lab execution by students is anticipated to last for about 2-3 hours. An in-classroom setup is envisaged, in order for tutors to help the students to explain the results they obtain via the remote lab exercises. Individual students' executions with remote tutor support are only allowed for a few students with special privileges (e.g. student-entrepreneurs). Individual student executions at anytime and anyplace will be open to all students in a next phase to work towards an even more enhanced blended learning approach. To serve the inclassroom lab execution to 90 students, we allow the lab to be taught in multiple lab sessions, to accommodate a subset of the student group in each session.

Identifying FIRE facilities. For this WLAN lab, the 'iMinds w-iLab.t' ${ }^{15}$ has been chosen as it provides full control over IEEE802.11 experimentation nodes (i.e. the technology needed for WLAN) with appropriate management and support. The 'iMinds Virtual Wall' has been used in addition, to host the educational content and to control the wireless experiment. Due to the nature of IEEE802.11, regardless of the FIRE facility, only 13 non-interfering radio channels can be used simultaneously (in the $5 \mathrm{GHz}$ spectrum) and hence only 13 experimental setups can be made available simultaneously. The FORGE widgets and FIRE adapters need to reckon for this fact by allowing the sharing of the same experimental setup amongst multiple student groups simultaneously. The

\footnotetext{
15 http://ilabt.iminds.be/testbeds
}

'iMinds w-iLab.t' provides furthermore a reservation mechanism, which guarantees the availability of the required experimentation nodes on the envisaged timeslots for inclassroom tutored sessions, as well as for remote sessions for a limited number of students.

Authoring educational content. For authoring the educational content, we could largely fall back to the material which already existed for an in-house lab about WLAN where we made use of a dedicated hardware setup which had to be cumbersomely configured and dismantled every year again and which could not be used by other institutions. The educational content itself (apart from the accompanying hardware setup) however has been fine-tuned over several years in order to match the level of student knowledge. Based on this content, we have deducted 10 different scenarios with 15 different questions for students to reflect on. As stated in the first step in the methodology (course requirements and learning outcomes), this selection and reformatting of the material has been done in order for the students to be able to focus on understanding the WLAN concepts and explaining WLAN throughput results, instead of consuming unnecessary time in configuring hardware.

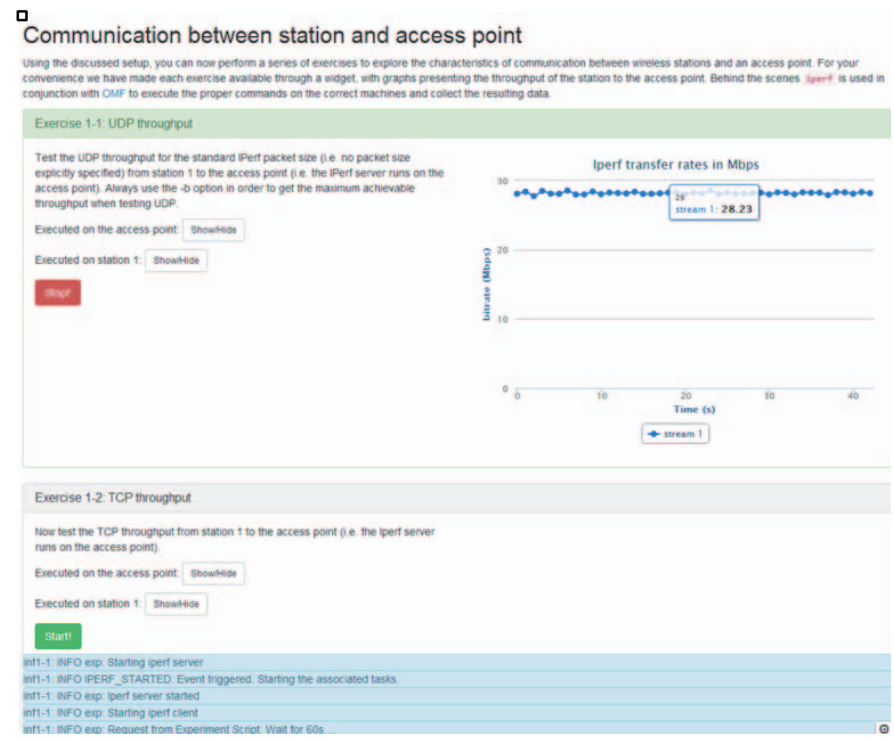

Figure 3: A screenshot of the interactive widget used by students in the WLAN lab in order to experiment with the iMinds w-iLab.t facility.

Integration of FIRE facilities and content. To run the 10 scenarios on the FIRE facility and to allow the simultaneous usage of the same resources by different student groups (without mutual interference), different widgets and FIRE adapters were created via different technologies while ensuring an easy-to-use, responsive and visually appealing web interface. Furthermore, the necessary e-learning tools had to be integrated, such as the coupling of students' actions to log entries in a Learning Analytics record store. Apart from these widgets for the actual student involvement, some widgets were also developed for the sake of teacher's convenience, such as 
an interface for the reservation of the resources on the intended time slots for the lab sessions.

Deployment. In February 2015, the WLAN lab with remote experimentation was deployed for 88 students. There was one in-classroom session for 45 students, a second in-classroom session for 41 students and a remote session for 2 students. Students were working in groups of two students each. We thus had a maximum of 23 students groups working simultaneously. Given the availability of 13 experimentation setups, this results in an acceptable overscription factor of 1.8 student groups per setup. In practice, this means that a student group will be able to execute a scenario experiment immediately or they will have to wait at the worst case for another group to finish their experiment (maximum 2 minutes). For the execution medium, a dedicated web site was used $^{16}$, with an offline paper answer sheet, which was corrected and marked by the tutors afterwards.
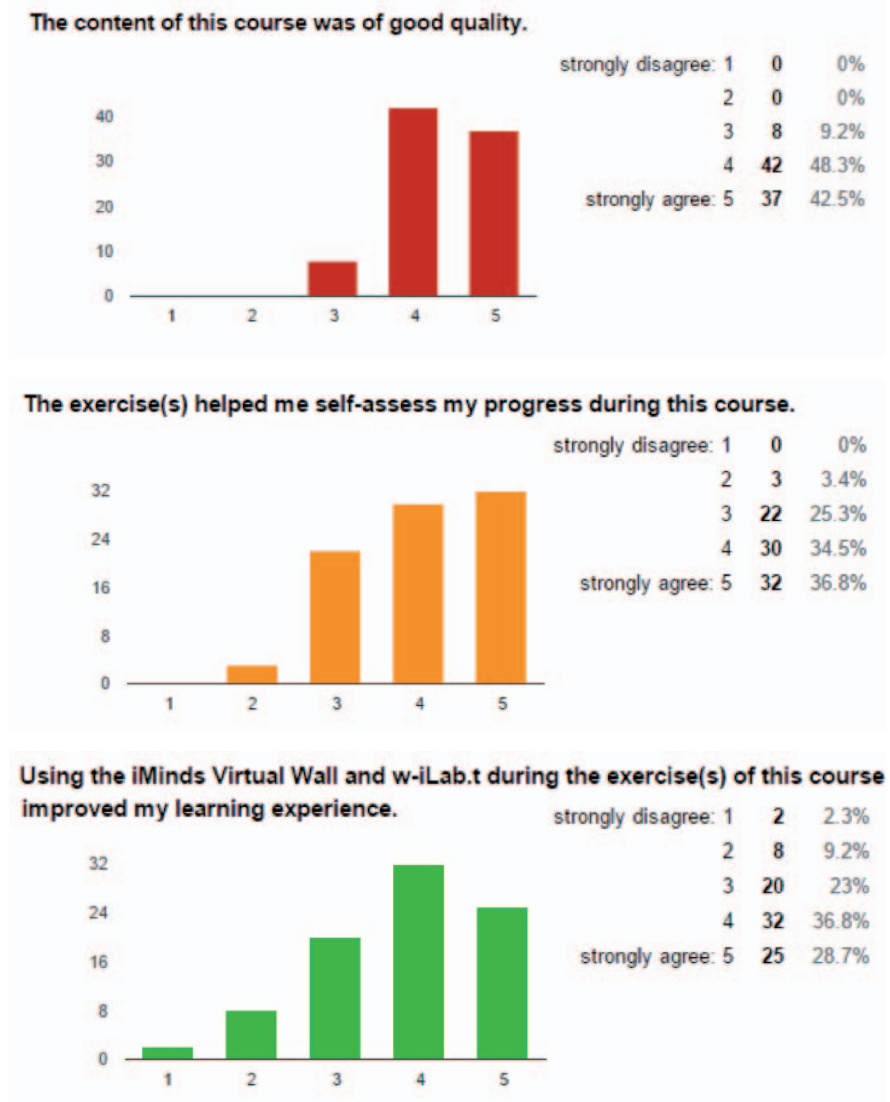

Figure 4: Student responses to the questionnaires used for the evaluation of the WLAN lab.

Evaluation. The students were asked to complete an online questionnaire immediately after finishing the lab session (with anonymous data processing clearly mentioned to the students). The questionnaire contains a few demographic questions (gender, age, enrolment program), 14 questions on a 5-Likert

${ }^{16} \mathrm{http}$ ///forge.test.iminds.be/wlan/ scale, 2 open questions and 1 question for remaining comments. All students were kind enough to complete the questionnaire. Furthermore, via the Learning Analytics implementation, which was integrated into the widgets, their actions were logged into the open source Learning Locker ${ }^{17}$ Learning Record Store. This will be post-processed to identify 'common mistakes' so we can focus on explaining the related aspects in next executions.

Reflection and adaptation. The questionnaire results showed great enthusiasm by the students undertaking the lab. Some frequent student remarks have been taken into consideration for next year's execution runs, e.g. online answer sheets instead of paper answer sheets. The exciting thing about developing a lab in this way is that it allows it to be taught in any part of the world at any time. After being developed and executed at a university in Belgium, the lab has been reused and taught in Ireland and Brazil as well, now totalling more than 135 involved students.

\section{B. Complying to the FORGE reference architecture}

The widgets and FIRE adapters which were developed in step 'Integration of FIRE facilities and content' were implemented following the reference architecture, as described in section III. All elements which were appropriate for the development of this specific WLAN lab are indicated in green in Figure 5. The widgets FIRE adapters e.g. ensured the sharing of a hardware setup by multiple student groups and for load balancing the student groups over all available hardware setups. The technical details are described in the project deliverables $^{18}$. The developments always kept platform independency in mind, in order to include these widgets in different media: dedicated web site for both large or small student devices (e.g. desktops, laptops, tablets, smartphones etc.), integration in an LMS, integration in eBooks, etc.

\section{CONCLUSIONS}

FORGE aims at complementing online learning initiatives with laboratory courses for an in-depth and hands-on educational experience. Within FORGE, our constructivist approach is based upon the notion of the experiment. FORGE allows students to create and conduct experiments using interactive learning resources within a comprehensive learning context. Towards this goal, FORGE has established a technological and pedagogical framework for remote labs and online experimentation, by defining a methodology for the design, delivery and evaluation of FIRE-enabled courseware.

Our next steps will be focused on the refinement of our methodology and its application in various learning contexts, especially in Higher Education. Other universities are currently integrating into their courses the lab described in this paper, as well as similar labs based on FIRE facilities. The deployment of our methodology in these contexts will provide us with valuable insights into the teaching and learning challenges faced by educators and learners respectively,

\footnotetext{
${ }^{17} \mathrm{http}: / /$ learninglocker.net

18 http://ict-forge.eu/wp-content/uploads/2015/02/FORGE-2014-PD311-Final.pdf and http://ict-forge.eu/wpcontent/uploads/2015/02/FORGE-2014-P-D21-Final.pdf
} 
regarding the use of remote labs for online experimentation. In order to identify and analyse these challenges, we will be deploying Learning Analytics methodologies as additional quantitative data collection instruments, complementing our qualitative data collection instruments of questionnaires and interviews.

\section{REFERENCES}

[1] D. E. Atkins, J. S. Brown, and A. L. Hammond, "A Review of the Open Educational Resources (OER) Movement: Achievements, Challenges, and New Opportunities," The William and Flora Hewlett Foundation 2007.

[2] J. Marquez-Barja, G. Jourjon, A. Mikroyannidis, C. Tranoris, J. Domingue, and L. DaSilva, "FORGE: Enhancing elearning and research in ICT through remote experimentation," in Proc. IEEE Global Engineering Education Conference (EDUCON), Istanbul, Turkey, 2014.

[3] T. De Jong, "Scaffolds for computer simulation based scientific discovery learning," in Dealing with complexity in learning environments, J. Elen and R. E. Clark, Eds. London: Elsevier Science Publishers, 2006, pp. 107-128.
[4] K. Hakkarainen, "Emergence of Progressive-Inquiry Culture in Computer-Supported Collaborative Learning," Science and Education, vol. 6, pp. 199-220, 2003.

[5] E. Kasl and L. Yorks, "Collaborative inquiry for adult learning," New Directions for Adult and Continuing Education, vol. 2002, pp. 312, 2002.

[6] R. Bose, "Virtual Labs Project: A Paradigm Shift in InternetBased Remote Experimentation," Access, IEEE, vol. 1, pp. 718-725, 2013.

[7] S. Frerich, D. Kruse, M. Petermann, and A. Kilzer, "Virtual Labs and Remote Labs: Practical experience for everyone," in Proc. IEEE Global Engineering Education Conference (EDUCON), Istanbul, Turkey, 2014, pp. 312-314.

[8] J. M. Marquez-Barja, N. Kaminski, F. Paisana, C. Tranoris, and L. A. DaSilva, "Virtualizing testbed resources to enable remote experimentation in online telecommunications education," in Proc. IEEE Global Engineering Education Conference (EDUCON), Tallinn, Estonia, 2015, pp. 836-843.

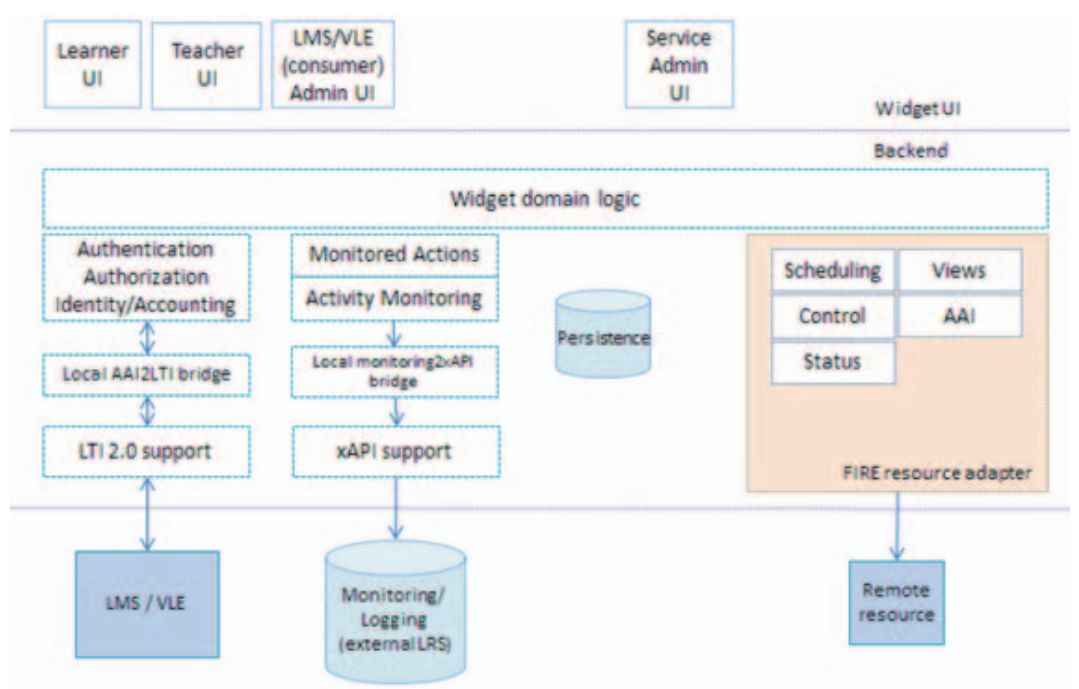

Figure 2: The FORGE widget reference architecture.

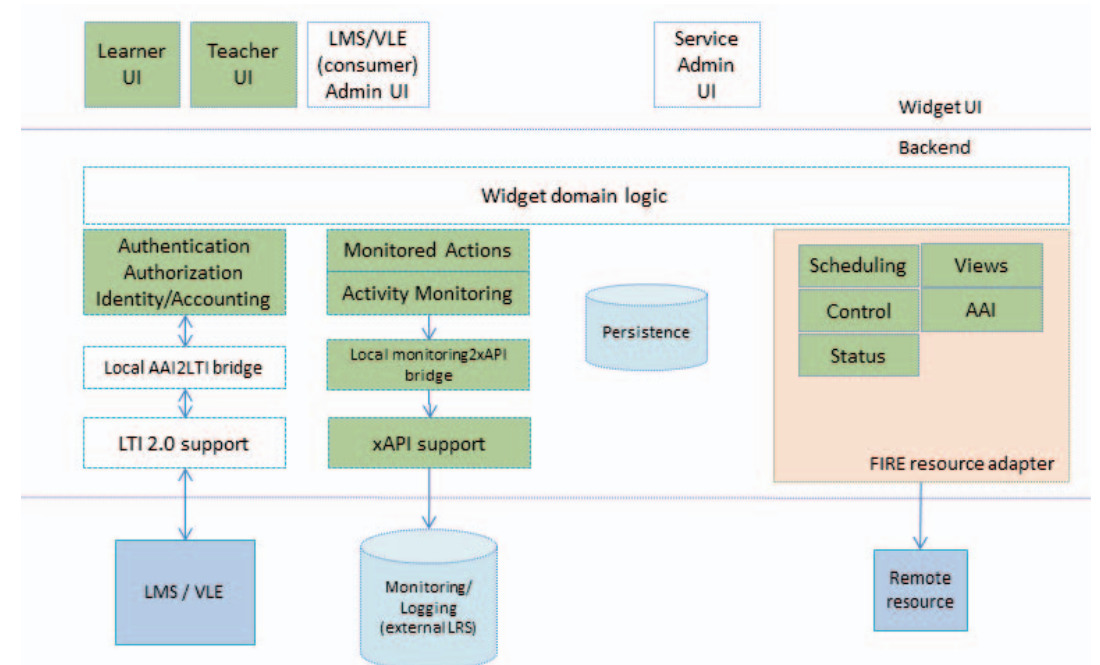

Figure 5: Using the FORGE widget reference architecture in the WLAN lab (deployed components are highlighted in green). 